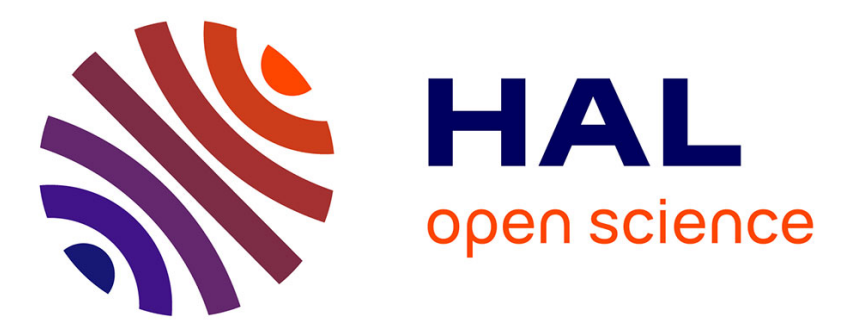

\title{
Direct mid-infrared femtosecond pulse shaping with a calomel acousto-optic programmable dispersive filter
}

Raman Maksimenka, Patrick Nuernberger, Kevin F. Lee, Adeline Bonvalet, Jadwiga Milkiewicz, Cestmir Barta, Milos Klima, Thomas Oksenhendler, Pierre Tournois, Daniel Kaplan, et al.

\section{To cite this version:}

Raman Maksimenka, Patrick Nuernberger, Kevin F. Lee, Adeline Bonvalet, Jadwiga Milkiewicz, et al.. Direct mid-infrared femtosecond pulse shaping with a calomel acousto-optic programmable dispersive filter. Optics Letters, 2010, 35 (21), pp.3565. 10.1364/OL.35.003565 . hal-00805067

\section{HAL Id: hal-00805067 \\ https://hal-polytechnique.archives-ouvertes.fr/hal-00805067}

Submitted on 9 May 2014

HAL is a multi-disciplinary open access archive for the deposit and dissemination of scientific research documents, whether they are published or not. The documents may come from teaching and research institutions in France or abroad, or from public or private research centers.
L'archive ouverte pluridisciplinaire HAL, est destinée au dépôt et à la diffusion de documents scientifiques de niveau recherche, publiés ou non, émanant des établissements d'enseignement et de recherche français ou étrangers, des laboratoires publics ou privés. 


\title{
Direct mid-infrared femtosecond pulse shaping with a calomel acousto-optic programmable dispersive filter
}

\author{
Raman Maksimenka, ${ }^{1, *}$ Patrick Nuernberger, ${ }^{2,3}$ Kevin F. Lee, ${ }^{2,3}$ Adeline Bonvalet, ${ }^{2,3}$ Jadwiga Milkiewicz, ${ }^{1}$ \\ Cestmir Barta, ${ }^{4}$ Miloš Klima, ${ }^{4}$ Thomas Oksenhendler, ${ }^{1}$ Pierre Tournois, ${ }^{1}$ \\ Daniel Kaplan, ${ }^{1}$ and Manuel Joffre ${ }^{2,3}$ \\ ${ }^{1}$ FASTLITE, Centre Scientifique d'Orsay-Bât. 503, 91401 Orsay, France \\ ${ }^{2}$ Laboratoire d'Optique et Biosciences, Ecole Polytechnique, CNRS, 91128 Palaiseau, France \\ ${ }^{3}$ Institut National de la Santé et de la Recherche Médicale, U696, 91128 Palaiseau, France \\ ${ }^{4}$ BBT Materials Processing, Doubicka 11, 18400 Prague, Czech Republic \\ *Corresponding author: raman@fastlite.com
}

Received July 26, 2010; revised September 16, 2010; accepted September 25, 2010; posted September 29, 2010 (Doc. ID 132222); published October 19, 2010

\begin{abstract}
Direct amplitude and phase shaping of mid-infrared femtosecond pulses is realized with a calomel-based acoustooptic programmable dispersive filter transparent between 0.4 and $20 \mu \mathrm{m}$. The shaped pulse electric field is fully characterized with high accuracy, using chirped-pulse upconversion and time-encoded arrangement spectral phase interferometry for direct electric field reconstruction techniques. Complex mid-infrared pulse shapes at a center wavelength of $4.9 \mu \mathrm{m}$ are generated with a spectral resolution of $14 \mathrm{~cm}^{-1}$, which exceeds by a factor of 5 the reported experimental resolutions of calomel-based filters. (c) 2010 Optical Society of America

OCIS codes: $\quad 160.1050,230.1040,320.5540$.
\end{abstract}

Recent developments of ultrafast mid-infrared (MIR) laser sources and pulse-shaping devices make a variety of new spectroscopic applications possible (see, e.g., discussion in $[1,2])$. For example, direct MIR pulse shaping was shown with a germanium acousto-optic modulator (Ge-AOM) integrated into a zero-dispersion optical delay line $[3,4]$, and its applicability to ground-state coherent control and two-dimensional MIR spectroscopy has been demonstrated [2].

Here, we present an alternative method of direct pulse shaping in the MIR domain, which is based on acoustooptic programmable dispersive filter (AOPDF or Dazzler) technology previously available only for near-infrared (NIR) [5], visible (VIS) [6], and UV [7]. Extension to the MIR has been proposed recently by Tournois [8]. This extension was made possible by advances in growing and processing of mercury halide materials, such as calomel [9-11].

AOPDFs are based on the acousto-optic effect in a collinear-beam geometry to selectively diffract spectral components of the incoming ultrashort pulse with desired group delays and amplitudes [12]. The advantage of this geometry is an extended interaction length and thus a high diffraction efficiency, a wide time aperture, and a high spectral resolution $[13,14]$.

The time aperture $\tau_{\max }$ of an $\overline{\mathrm{AO}} \overline{\mathrm{PD}} \mathrm{F}$ pulse shaper can be expressed in the following way:

$$
\tau_{\max }=\frac{L\left(n_{g d}-n_{g i}\right)}{c},
$$

where $L$ is the interaction length, $n_{g i}$ and $n_{g d}$ are the group indices of the incident and diffracted waves, respectively, and $c$ is the speed of light in vacuum.

The spectral resolution of an acousto-optic device is related to the time aperture by the time-bandwidth product and is given by [12]

$$
\delta \tilde{\nu}=\frac{0.8}{c \tau_{\max }},
$$

where $\delta \tilde{\nu}$ is the minimum linewidth in $\mathrm{cm}^{-1}$.

Paratellurite crystals employed in VIS and NIR AOPDFs are limited by their transparency range to wavelengths below $4 \mu \mathrm{m}$. Calomel (mercurous chloride, $\mathrm{Hg}_{2} \mathrm{Cl}_{2}$ ) has a transparency range between 0.4 and $20 \mu \mathrm{m}$, a high acousto-optic figure of merit, a low acoustic velocity, and a high birefringence [9]. It is, thus, an ideal candidate for an AOPDF in the MIR range. We report the realization and preliminary experimental demonstration of such a device. Although acousto-optic filters using calomel have been previously reported [11], this is, to the best of our knowledge, the first report of a calomel AOPDF, i.e., using the collinear-beam geometry for ultrafast pulse shaping.

The experimental setup is sketched in Fig. 1. Pulses with a spectral FWHM of $0.3 \mu \mathrm{m}$ centered at $4.9 \mu \mathrm{m}$

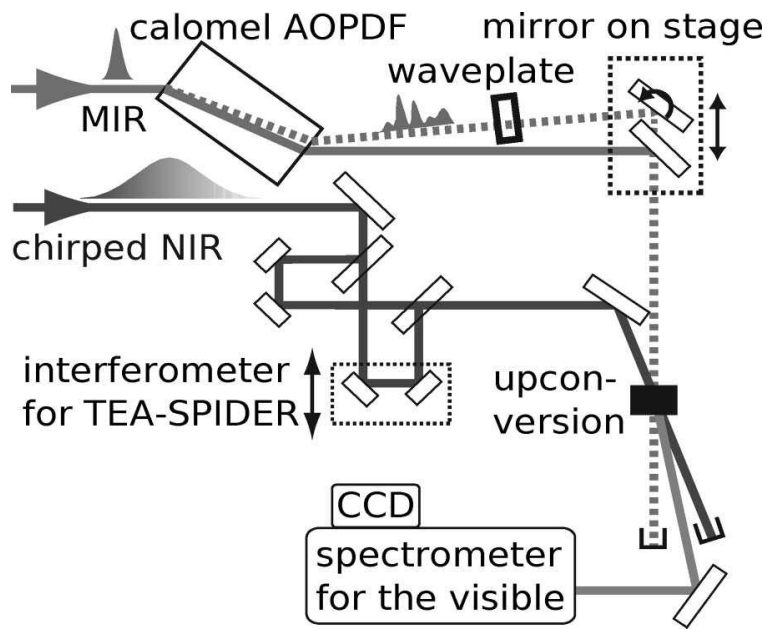

Fig. 1. Experimental setup. 
are generated in a two-stage optical parametric amplifier with subsequent difference frequency mixing. The MIR pulses traverse the calomel crystal along with an acoustic wave shaped by an arbitrary wave-function generator. Both the optical and the acoustic beam have a diameter of $2 \mathrm{~mm}$ and propagate collinearly in the crystal at an angle of $17.3^{\circ}$ to the optical axis. The central frequency of the acoustic wave corresponding to an optical wavelength of $4.9 \mu \mathrm{m}$ is $6.8 \mathrm{MHz}$. The propagation length in the crystal is $18.3 \mathrm{~mm}$, yielding acoustic and optical time windows of $22.5 \mu \mathrm{s}$ and $2.5 \mathrm{ps}$, respectively, which allows for the programmable group-velocity dispersion of up to $10^{4} \mathrm{fs}^{2}$ for the pulses used in the experiment. This particular geometry was chosen to minimize losses due to reflection, which are about $14 \%$ at the input face $(s$ polarized) and $5 \%$ at the output face ( $p$ polarized). In the future, use of antireflection coatings will reduce optical losses to a few percent. The acoustic propagation time of $22.5 \mu \mathrm{s}$ limits the maximum repetition rate of the AOPDF operation to $44 \mathrm{kHz}$.

The nondiffracted and diffracted optical beams are separated by an angle of $4.7^{\circ}$ at the output of the crystal, allowing geometrical separation of the diffracted beam without resorting to polarization selectivity. Either the transmitted (for calibration purposes) or the diffracted beam (whose polarization is perpendicular to the one of the transmitted beam and is rotated back by a tunable MIR wave plate) is upconverted with a chirped $800 \mathrm{~nm}$ pulse to the VIS spectral regime and analyzed in a spectrometer with a CCD camera (Fig. 1) [15]. The shaped MIR pulses are fully characterized by time-encoded arrangement spectral phase interferometry for direct electric field reconstruction (TEA-SPIDER) [16]. The MIR spectra are corrected for the chirp of the $\overline{80} 0 \mathrm{~nm}$ pulse; calibration is done directly in the MIR using narrow atmospheric absorption lines [17].

With a monochromatic acoustic wave, a single-point diffraction efficiency of $40 \%$ is observed [Fig. 2(a)]. The corresponding minimum spectral resolution for the diffracted pulses is $14 \mathrm{~cm}^{-1}$ FWHM in intensity, which approaches the theoretical value of $11 \mathrm{~cm}^{-1}$. This result is an improvement of more than a factor of 5 with respect to previously reported performances of calomel-based conventional acousto-optic filters that did not incorporate the collinear-beam configuration design [11]. Figure 2(b) shows diffracted spectra, each one obtained with a narrowband Gaussian-shaped acoustic wave at a different frequency (solid curves) superimposed with the diffracted spectrum obtained with a broadband acoustic pulse (dashed curve, magnified 17 times). Gaussianshaped acoustic pulses are used here instead of monochromatic acoustic waves in order to increase the spectral contrast of the isolated pulses at the expense of the spectral resolution. Narrow spectral dips correspond to water vapor absorption [17]. FWHM of the observed spectra is about $16 \mathrm{~cm}^{-1}$.

If two identical but temporally delayed acoustic pulses are applied, two delayed optical pulses are generated and their spectrum exhibits spectral interference. Figure 2(c) shows the spectral fringes resulting from the interference of two diffracted pulses created by an acoustic double pulse separated by $20 \mu \mathrm{s}$ (corresponds to $2.22 \mathrm{ps}$ optical delay). One can distinguish 23 peaks within a $30 \mathrm{~dB}$ dy-
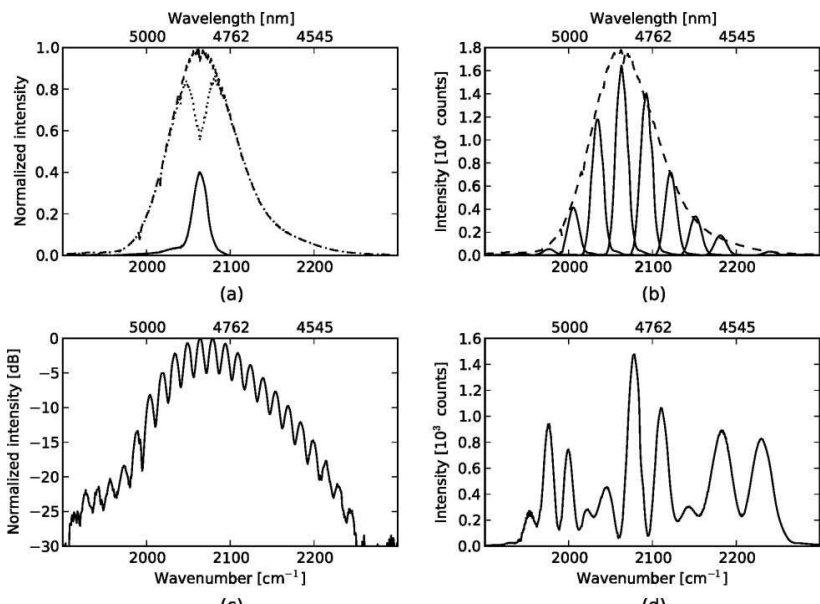

Fig. 2. Examples of amplitude shaping: (a) spectra of the transmitted MIR pulse with acoustic wave on (dotted curve, $I_{\text {on }}$ ) and off (dashed curve, $I_{\text {off }}$ ) and the corresponding relative difference (solid curve, $\left.\left(I_{\text {off }}-I_{\text {on }}\right) / I_{\text {off }}\right)$; (b) broadband (dashed curve, $\delta \nu_{\mathrm{FWHM}}^{a c}=0.7 \mathrm{MHz}$ ) and a series of narrowband (solid curves, $\delta \nu_{\text {FWHM }}^{a c}=0.03 \mathrm{MHz}$ ) diffraction spectra; (c) spectral interference pattern for double pulse (note the logarithmic $y$ scale); (d) spectral interference pattern for three narrowband pulses with different central frequencies and one broadband pulse.

namic range $\left(1: 10^{-3}\right)$ spanning through $340 \mathrm{~cm}^{-1}$, thus yielding a resolution of $14 \mathrm{~cm}^{-1}$. To illustrate the amplitude-shaping capability, a spectrally separated pulse sequence is created: a broadband acoustic pulse that diffracts all MIR frequencies is followed by three acoustic pulses delayed by $13 \mu \mathrm{s}, 9 \mu \mathrm{s}$, and $5 \mu \mathrm{s}$, which diffract only the low-, middle-, and high-frequency parts of the spectrum, respectively. Thus, an interference pattern arises for which the fringe spacing is different in the three spectral regions [see Fig. 2(d)].

The pulse-shaping capacity of the AOPDF is limited to the available time window, that is to $2.5 \mathrm{ps}$ for the device used here. A series of phase-modulated acoustic waves is loaded into the calomel crystal in order to quantitatively compare the programmed phase to phase measurements. Calibration of the acoustic-to-optic frequency ratio required for calculation of polynomial phase coefficients was performed by scanning the acoustic frequency and detecting the corresponding frequency of the diffracted light [see, e.g., Fig. 2(b)]. Experimental data obtained for polynomial spectral phase functions are presented in Fig. 3, with an intensity-weighted mean group delay and an absolute phase at central frequency subtracted from both applied and measured phase functions. The desired optical phase encoded into the acoustic wave agrees well with the phase determined by TEA-SPIDER. The phase error is calculated as an rms of the variation of phase difference weighted by the spectral density. The quality of the phase reconstruction was verified by the accuracy criterion $(\varepsilon>0.1 \mathrm{rad}$ is "poor" and $\varepsilon<$ $0.02 \mathrm{rad}$ is "very good") derived in [18], yielding excellent results for MIR pulse shaping, as evidenced by the corresponding $\varepsilon$ values given in Fig. 3 .

The key advantages of the AOPDF pulse shapers are their simplicity and compactness, as well as absence of angular spatio-temporal coupling. The footprint of 


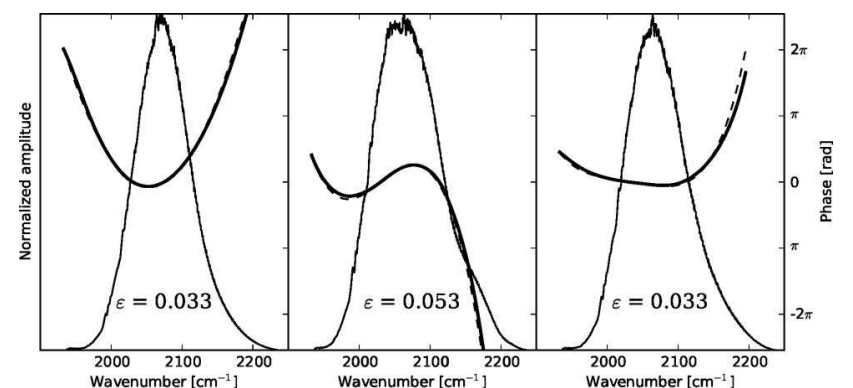

Fig. 3. Examples of phase modulations induced by the AOPDF and characterized with TEA-SPIDER. From left to right: quadratic, cubic, and fourth-order applied (dashed lines) and measured (solid lines) phases. Phase error $\varepsilon$ is displayed for each phase function. The spectra of the diffracted pulses are shown in the background.

the mounted acousto-optic crystal and accompanying electric matching circuit is only 4 by $6 \mathrm{~cm}$. It is inserted directly in the optical setup into the MIR beam path and requires minimum alignment.

Pulse-shaping capabilities of the device tested here are somewhat inferior to those reported for Ge-AOM [2]; e.g., the experimentally observed optical time window is only $2.2 \mathrm{ps}$ for the calomel-AOPDF, whereas it is $13 \mathrm{ps}$ for the Ge-AOM-based shaper. The same is true for the resolution: about $30 \mathrm{~nm}$ at $4.9 \mu \mathrm{m}$ for the calomel-AOPDF and $5 \mathrm{~nm}$ for the Ge-AOM-based shaper. For comparison, the resolution demonstrated by the same authors for the pulse shaping via parametric transfer is $50 \mathrm{~nm}$ at $4.9 \mu \mathrm{m}$ [2]. It should be noted, however, that the full potential provided by the extreme birefringence of calomel single crystal $\left(n_{g e}-n_{g o}\right.$ up to 0.55 at $\left.4.9 \mu \mathrm{m}\right)$ was not exploited in the present work, and the corresponding experiments are underway now.

Taking into account the reflection and absorption losses, single-point throughput of the calomel-AOPDF is about $30 \%$, which is comparable to the performance reported for the Ge-AOM-based shaper [3]. Unlike the zero-dispersion line-based shapers, AOPDF diffraction efficiency scales inversely with the number of diffracted points. This effect could be compensated by increasing the applied acoustic power, as is done in commercially available devices for the VIS and NIR range. In the device tested here, however, the maximum acoustic power in the crystal was not sufficient to increase diffraction efficiency; hence, the total throughput of the MIR pulse (340 $\mathrm{nm}$ or 23 resolution points) was only about $1 \%$.
In conclusion, direct amplitude and phase shaping of ultrashort MIR pulses by means of a calomel-AOPDF was demonstrated. Complex pulse shapes were generated and fully characterized with high accuracy, exemplifying the versatility of the device. Technological studies are in progress to improve the diffraction efficiency and the spectral resolution. Prospective applications comprise selective ground-state photochemistry, two-dimensional MIR spectroscopy, or even the manipulation of MIR near-field effects in nanophotonics.

This work was supported by Eurostars Program E! (244), the MIRSA, Délégation Générale de l'Armement REI Program (2008 34 0001), the European ITN Program 214962-FASTQUAST, the Agence Nationale de la Recherche (ANR-06-BLAN-0286), the Conseil Général de l'Essonne (Astre 2006), and the Deutsche Akademie der Naturforscher Leopoldina (BMBF-LPDS 2009-6).

\section{References}

1. C. Erny, L. Gallmann, and U. Keller, Appl. Phys. B 96, 257 (2009).

2. D. B. Strasfeld, S.-H. Shim, and M. T. Zanni, Adv. Chem. Phys. 141, 1 (2009).

3. S.-H. Shim, D. B. Strasfeld, E. C. Fulmer, and M. T. Zanni, Opt. Lett. 31, 838 (2006).

4. M. Tsubouchi and T. Momose, Opt. Commun. 282, 3757 (2009).

5. F. Verluise, V. Laude, Z. Cheng, C. Spielmann, and P. Tournois, Opt. Lett. 25, 575 (2000).

6. A. Monmayrant, A. Arbouet, B. Girard, B. Chatel, A. Barman, B. J. Whitaker, and D. Kaplan, Appl. Phys. B 81, 177 (2005).

7. S. Coudreau, D. Kaplan, and P. Tournois, Opt. Lett. 31 , 1899 (2006).

8. P. Tournois, Opt. Commun. 281, 4054 (2008).

9. C. Barta, Krist. Tech. 5, 541 (1970).

10. C. Barta, J. Cryst. Growth 65, 351 (1983).

11. M. Gottlieb, A. Goutzoulis, and N. Singh, Appl. Opt. 26, 4681 (1987).

12. D. Kaplan and P. Tournois, J. Phys. IV 12, 69 (2002).

13. I. C. Chang, Electron. Lett. 28, 1255 (1992).

14. V. B. Voloshinov, Opt. Eng. 31, 2089 (1992).

15. K. J. Kubarych, M. Joffre, A. Moore, N. Belabas, and D. M. Jonas, Opt. Lett. 30, 1228 (2005).

16. K. F. Lee, K. J. Kubarych, A. Bonvalet, and M. Joffre, J. Opt. Soc. Am. B 25, A54 (2008).

17. K. F. Lee, P. Nuernberger, A. Bonvalet, and M. Joffre, Opt. Express 17, 18738 (2009).

18. C. Dorrer and I. A. Walmsley, J. Opt. Soc. Am. B 19, 1019 (2002). 